\title{
X-ray observations of HESS J1702-420 and HESS J1427-608 with Suzaku
}

\section{Takahisa Fujinaga*}

Institute of Space and Astronautical Science/JAXA,

3-1-1 Yoshinodai, Chuo-ku, Sagamihara, Kanagawa 252-5210, JAPAN;

Tokyo Institute of Technology

2-12-1-H-36 Ookayama, Meguro-ku, Tokyo 152-8550, JAPAN

E-mail: fujinaga@astro.isas.jaxa.jp

Aya Bamba ${ }^{1,2}$, Koji Mori ${ }^{3}$, Tadayasu Dotani ${ }^{2,4}$, Masanobu Ozaki ${ }^{4}$, Shoichi Kimura ${ }^{3}$, Hironori Matsumoto ${ }^{5}$, Yukikatsu Terada ${ }^{6}$, Hideki Uchiyama ${ }^{7}$, Gerd Pühlhofer ${ }^{8}$, Stefan Wagner ${ }^{9}$, Olaf Reimer ${ }^{10,11}$, Stefan Funk ${ }^{11}$, Jim Hinton ${ }^{12}$, Karl Kosack ${ }^{13}$, Nukri Komin $^{13}$, and Jean-Francois Glicenstein ${ }^{13}$

${ }^{1}$ Dublin Institute for Advanced Studies, ${ }^{2}$ Institute of Space and Astronautical Science/JAXA,

${ }^{3}$ University of Miyazaki, ${ }^{4}$ Tokyo Institute of Technology, ${ }^{5}$ Nagoya University,

${ }^{6}$ Saitama University, ${ }^{7}$ The University of Tokyo,

${ }^{8}$ Institut für Astronomie und Astrophysik, Universität Tübingen,

${ }^{9}$ Landessternwarte, Universität Heidelberg, ${ }^{10}$ Leopold-Franzens-Universität Innsbruck,

${ }^{11}$ SLAC National Accelerator Laboratory, ${ }^{12}$ University of Leicester, ${ }^{13}$ IRFU/DSM/CEA

\begin{abstract}
X-ray follow-up observations of unidentified (unID) very high energy (VHE) gamma-ray sources, HESS J1702-420 and HESS J1427-608, are presented. HESS J1702-420 is one of the brightest unID sources in the VHE band. Despite of the deep observation with Suzaku, no new X-ray source was found in the field of view of X-ray Imaging Spectrometer (XIS). If we apply a simple one-zone model to compare the derived upper limit of X-ray flux, $F_{\mathrm{X}}<2.4 \times 10^{-12} \mathrm{erg} \mathrm{s}^{-1} \mathrm{~cm}^{-2}$ in the $2-10 \mathrm{keV}$ band and that in the $1-10 \mathrm{TeV}$ band, the magnetic field involved in the VHE gamma-ray emission region becomes rather low $(B<0.9 \mu \mathrm{G})$. HESS J1427-608 is one of the most compact unID sources in the VHE band with no associated pulsars or supernova remnants. The Suzaku observation revealed presence of a slightly extended source in the XIS field of view, which coincides with the center of the VHE gamma-ray source.
\end{abstract}

25th Texas Symposium on Relativistic Astrophysics - TEXAS 2010

December 06-10, 2010

Heidelberg, Germany

*acknowledges the financial support from the Global Center of Excellence Program by MEXT, Japan through the "Nanoscience and Quantum Physics" project of the Tokyo Institute of Technology. 


\section{Introduction}

The origin of cosmic rays is an unsolved problem since their discovery in the early 20th century. Direct evidence of accelerated electrons exceeding $\sim 1 \mathrm{TeV}$ was found at young supernova remnants (SNRs) in the X-ray band (e.g. [1]). However, any clear evidence of proton acceleration has not been obtained yet observationally.

Recently, High Energy Stereoscopic System (H.E.S.S.) performed survey observations along the Galactic plane, and found more than 50 new sources in the very high energy (VHE) gamma-ray band [2]. VHE gamma-rays are produced through either leptonic process, i.e. inverse Compton scattering of low energy photons (e.g. cosmic microwave background (CMB) or interstellar optical/infrared emission) by relativistic electrons, or hadronic process, i.e. the decay of pions produced by collisions of relativistic protons and interstellar matter. If relativistic electrons are present, they may be traced through the observations of synchrotron X-ray emission. Thus, X-ray follow-up observations enable us to distinguish the origin of VHE gamma-ray emission from the recently discovered Galactic VHE sources. In fact, two thirds of the sources were found to be associated with SNRs or pulsar wind nebulae (PWNe). For most of the remaining sources, no counter parts have been found in other wavebands, and their nature is still unclear. These sources are often referred to as unidentified (unID) sources, or as "dark" particle accelerators ([3]).

We observed two unID sources whose nature is still unknown, HESS J1702-420 and HESS

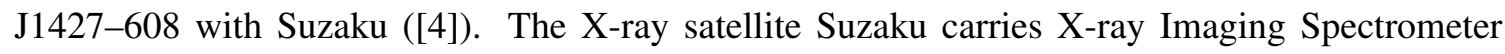
(XIS: [5]), which covers $17^{\prime} .8 \times 17^{\prime} .8$ field of view (FOV) and can take spectroscopic data in the $0.2-12 \mathrm{keV}$ band with charge coupled device (CCD). Suzaku is characterized with the low and stable background compared to other X-ray satellites which is useful to search for possible counterparts.

\section{HESS J1702-420}

\subsection{Properties in the VHE gamma-rays}

HESS J1702-420 is one of the brightest sources among the unID VHE sources, whose flux is $F_{\mathrm{TeV}}=3.1 \times 10^{-11} \mathrm{erg} \mathrm{s}^{-1} \mathrm{~cm}^{-2}$ in the $1-10 \mathrm{TeV}$ band with a photon index of $\Gamma_{\mathrm{TeV}}=2.1$ (《2]; [6). It is located at $l=344^{\circ} .26, b=-0^{\circ} .22$ and extends $15-30^{\prime}$ assymmetrically (see the green contour in Fig. 11. No known source exists within $15^{\prime}$ in any wavebands.

\subsection{X-ray observation and the results}

We performed a deep X-ray observation including the center of HESS J1702-420 on 25-30 March 2008. The effective exposure was $216 \mathrm{ks}$. To estimate the spectrum of the X-ray background, we also used nearby archive data of Suzaku, whose aim points were $l=332^{\circ} .00, b=-0^{\circ} .15$ and $l=332^{\circ} .40, b=-0^{\circ} .15$ respectively. The spaced-row charge injection (SCI: [7], 《8]) was used for the observation of HESS J1702-420 to reduce the effects of radiation damage, whereas the background observations are carried out with SCI-off. Because dead areas are introduced in the XIS image with SCI-on, they were taken into account of the response files. 


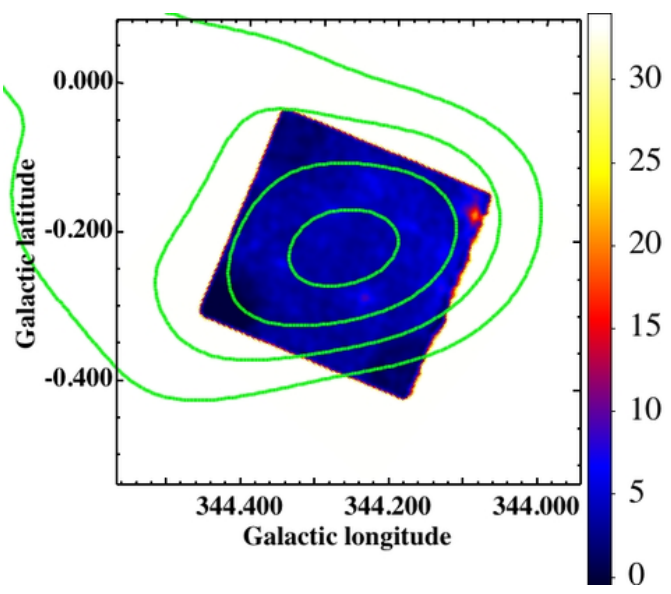

Figure 1: XIS image of HESS J1702-420 field in the $2-8 \mathrm{keV}$ band. The unit of the color scale is count pixel $^{-1}$, which the vignetting was corrected after the subtraction of NXB. The green contour shows the H.E.S.S. intensity map.

Figure 1 shows XIS image in the $2-8 \mathrm{keV}$ band, in which the vignetting is corrected after the subtraction of non X-ray background (NXB: [9]). No new X-ray bright emission was seen in the image.

Next, we look for a diffuse excess emission, which may be associated to HESS J1702-420. We assumed that the X-rays from HESS J1702-420 field is uniformly extended in the XIS FOV. The X-ray emission from the field of HESS J1702-420 is considered to be composed of the cosmic $\mathrm{X}$-ray background (CXB), Galactic ridge X-ray emission (GRXE: e.g. [10]), and the diffuse excess emission from HESS J1702-420. We modeled the X-ray spectrum of CXB+GRXE as a power-law and three gaussians, which correspond to the iron lines between 6 and $7 \mathrm{keV}$. These models and their parameters are commonly used among HESS J1702-420 and the background data. Also, we modeled the excess emission of HESS J1702-420 as a power-law with the assumed $\Gamma=2.1$. The excess emission was not significant, and an upper limit of the excess X-ray flux in the 2-10 keV band was $F_{\mathrm{X}}<2.4 \times 10^{-12} \mathrm{erg} \mathrm{s}^{-1} \mathrm{~cm}^{-2}$ in the $90 \%$ confidence limit.

\subsection{Discussion}

We could not detect any possible X-ray counterpart and derived an upper limit of the diffuse $\mathrm{X}$-ray flux. To estimate the physical parameters of the source, we evaluate a flux ratio $\left(F_{\mathrm{TeV}} / F_{\mathrm{X}}\right)$. Using the derived upper limit of the $\mathrm{X}$-ray flux, the ratio becomes large $\left(F_{\mathrm{TeV}} / F_{\mathrm{X}}>12\right)$. The lower limit of the ratio is comparable to known dark particle accelerators, such as HESS J1616-508 with $F_{\mathrm{TeV}} / F_{\mathrm{X}}>55([11])$ and HESS J1804-216 with $F_{\mathrm{TeV}} / F_{\mathrm{X}}>13$ ([12]). We conclude that HESS J1702-420 is another dark particle accelerator.

If we assume that the VHE gamma-rays are produced via the inverse Compton scattering of the CMB by electrons, and $\mathrm{X}$-rays via the synchrotron emission, the flux ratio corresponds to the ratio of the energy densities of $\mathrm{CMB}$ and the magnetic field. The X-ray spectrum can be estimated from the VHE gamma-ray spectrum assuming a simple one-zone model. The wide-band spectral energy distribution with the estimated X-ray spectra is shown in Figure 2 as a function of the magnetic 


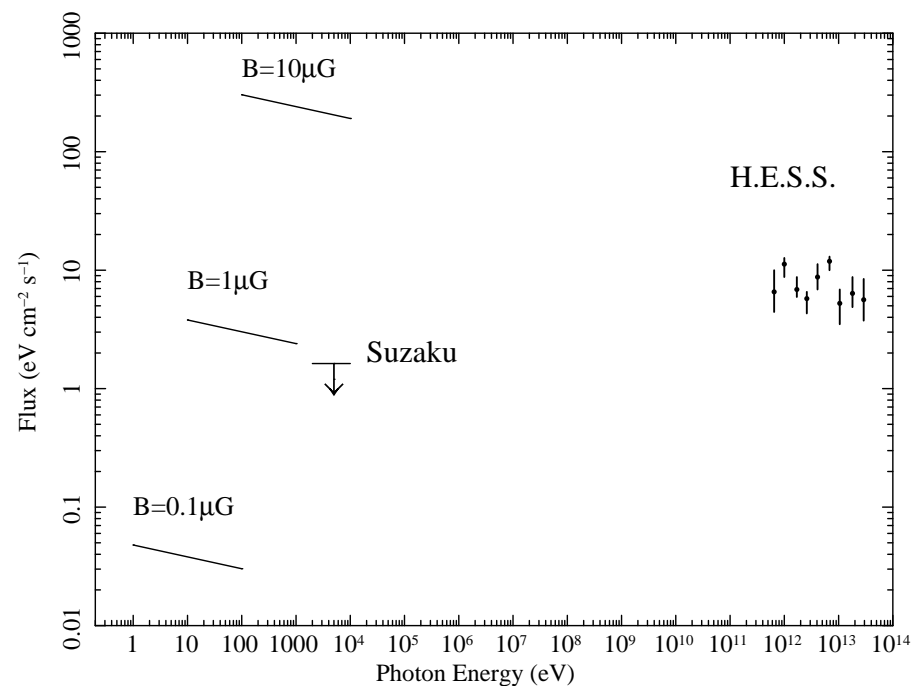

Figure 2: The wide-band spectrum of HESS J1702-420. The synchrotron X-ray emission expected for the one-zone model is indicated as a function of the magnetic field.

field. According to the derived flux ratio, the local magnetic field would be $B<0.9 \mu \mathrm{G}$, which is smaller than the typical value of $B \simeq 3-10 \mu \mathrm{G}$ on the Galactic plane. This result leads the following conjecture: (1) a simple one-zone model does not hold for HESS J1702-420, and (2) the VHE gamma-ray emission may be originated not from electrons but from protons.

\section{HESS J1427-608}

\subsection{Properties in the VHE gamma-rays}

HESS J1427-608 is located at $l=314^{\circ} .41, b=-0^{\circ} .15$. This is one of the most compact unID sources and its extension is $2.4-4.8$ arcmin. The flux in the $1-10 \mathrm{TeV}$ is $F_{\mathrm{TeV}}=2.5 \times$ $10^{-12} \mathrm{erg} \mathrm{s}^{-1} \mathrm{~cm}^{-2}$ with the photon index of $\Gamma=2.2(\llbracket 6)$. No known source exists within $30^{\prime}$ in any wavebands. Furthermore, because HESS J1427-608 is separated more than $100^{\prime}$ from the nearest energetic pulsar, it may not be a PWN.

\subsection{X-ray observation and the results}

To search for an associated X-ray emission, we observed the HESS J1427-608 field on 13-16 January 2010. The effective exposure was $104 \mathrm{ks}$.

A new X-ray source was detected in the XIS image at the center of FOV (see Figure 3). We fitted the one-dimensional profiles of the X-ray image along with the right ascension and declination with gaussian, and found the gaussian width was $\sigma=1^{\prime} .2-1^{\prime} .4$. The extent is a little larger than that of the point source derived from a simulation $\left(\sigma=0^{\prime} .9\right)$. Thus, the X-ray source is slightly extended.

Next, we extracted the spectrum of the X-ray source within $4^{\prime}$. The background was subtracted using the ring region surrounding it with a radius of $7^{\prime}$. We fitted the spectrum with an absorbed power-law in the $1.5-8.0 \mathrm{keV}$ band (see Figure 4). The best-fit parameters are as follows: 


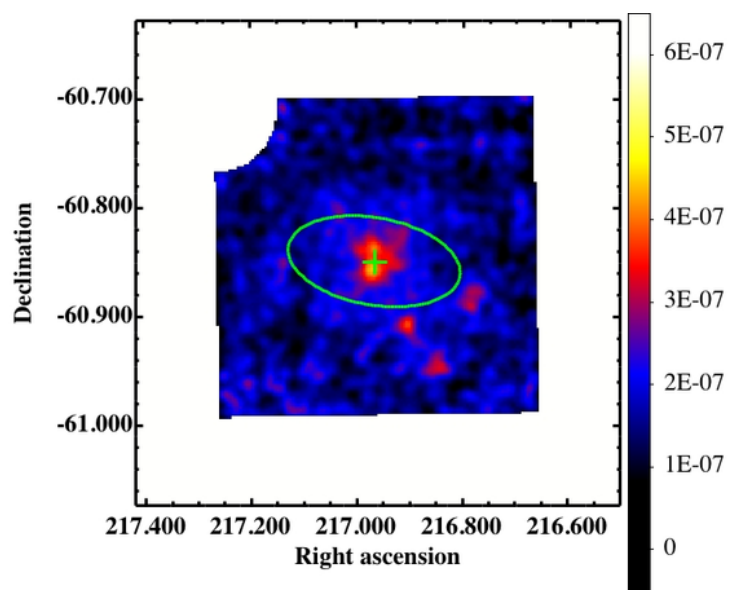

Figure 3: XIS image of HESS J1427-608 field in the $2-8 \mathrm{keV}$ band. The unit of the color scale is count $\mathrm{s}^{-1}$ pixel $^{-1}$, which the vignetting and exposure were corrected after the subtraction of NXB. The green ellipse indicates the $1 \sigma$ extent of VHE gamma-ray emission. The center of the VHE extension is showed by the green cross [6].

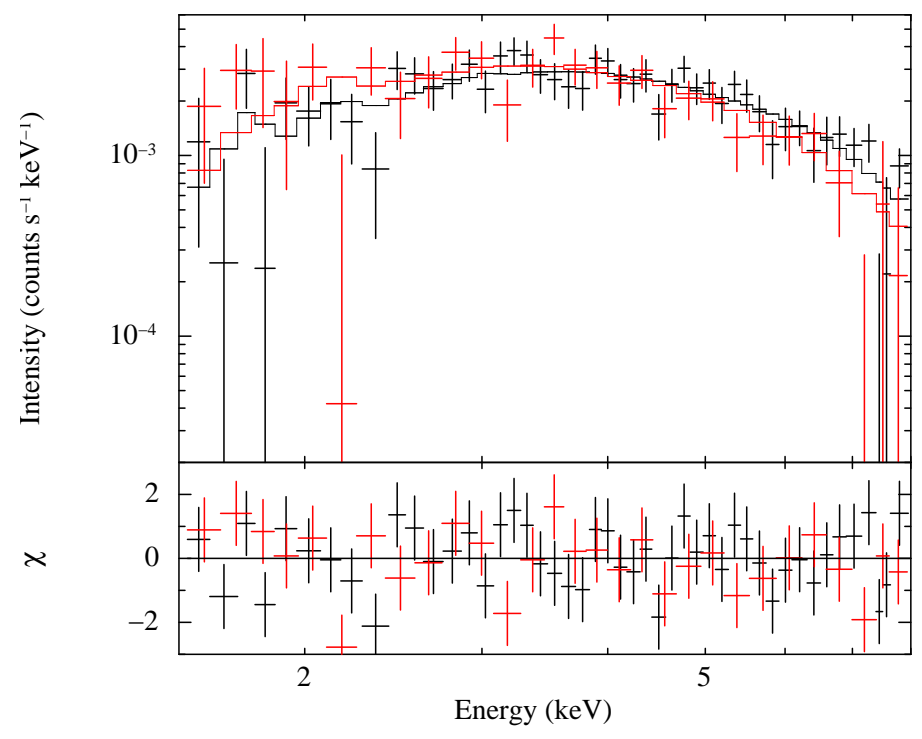

Figure 4: XIS spectra of the X-ray source in the $1.5-8.0 \mathrm{keV}$ band. Black and red indicate the frontilluminated and the back-illuminated CCDs, respectively.

$N_{\mathrm{H}}=5.1_{-1.1}^{+1.3}\left(10^{22} \mathrm{~cm}^{-2}\right), \Gamma=2.0_{-0.3}^{+0.4}$, and the flux $F_{\mathrm{X}}=3.1_{-0.3}^{+0.4}\left(10^{-12} \mathrm{erg} \mathrm{s}^{-1} \mathrm{~cm}^{-2}\right)$ in the $2-10 \mathrm{keV}$ band. The errors of each parameters are in the $90 \%$ confidence region, and the fit was acceptable with $\chi^{2} /$ d.o.f. $=75.34 / 73$.

\subsection{Discussion}

The position of the X-ray source coinsides with the VHE source, while its extent $\left(\sigma=1^{\prime} .2-\right.$ $\left.1^{\prime} .4\right)$ is smaller than that of the VHE source $\left(\sigma=2^{\prime} .4-4^{\prime} .8\right)$. The X-ray source may be a possible 
counterpart to HESS J1427-608. If we assume that the VHE gamma-rays are originated from the Compton up-scattered photons by energetic electrons, X-ray emission can be explained by synchrotron emission in a magnetic field of $B \simeq 3.7 \mu \mathrm{G}$. Thus, we could not distinguish whether relativistic protons or electrons emit the VHE gamma-rays in the case of HESS J1427-608.

Considering the position coincidence and the similarity with typical PWNe (e.g. the X-ray counterpart has an extended emission, and its photon index is $\Gamma \simeq 2.0$ ), a PWN is one of the possibilities of HESS J1427-608 despite of the fact that there is no bright nearby pulsars. According to [13], the characteristic age and X-ray luminosity can be estimated using the flux ratio $\left(F_{\mathrm{TeV}} / F_{\mathrm{X}} \simeq 0.8\right)$ to be $\tau \simeq 4.4 \mathrm{kyr}$ and $L_{\mathrm{X}} \simeq 4 \times 10^{34} \mathrm{erg} \mathrm{s}^{-1}$, respectively. Once the luminosity is estimated, we can derive the distance to HESS J1427-608 to be $d \simeq 10 \mathrm{kpc}$. However, available information on HESS J1427-608 is very limited and its nature is still unclear. Further studies are necessary to reveal its nature.

\section{Summary}

HESS J1702-420 and HESS J1427-608 are observed by Suzaku. Any significant X-ray emission was not found in the HESS J1702-420 field. This means that the VHE gamma-rays from HESS J1702-420 may be produced by relativistic protons. On the other hand, a slightly extended source was found at the center of HESS J1427-608. These results support that VHE unID sources have large varieties in nature, for example, "dark" particle accelerators, PWNe, or SNRs, etc. To understand the population of the whole unID sources, we need further X-ray observations for the sources which have not been observed yet in the X-ray band.

\section{References}

[1] Koyama, K., et al. 1995, Nature, 378, 255

[2] Aharonian, F., et al. 2006, ApJ, 636, 777

[3] Aharonian, F., et al. 2005, Science, 307, 1938

[4] Mitsuda, K., et al. 2007, PASJ, 59, S1

[5] Koyama, K., et al. 2007, PASJ, 59, S23

[6] Aharonian, F., et al. 2008, A\&A, 477,353

[7] Prigozhin, G., et al. 2008, IEEE Transactions on Electron Devices, 55, 2111

[8] Uchiyama, H., et al. 2009, PASJ, 61, S9

[9] Tawa, N., et al. 2008, PASJ, 60, S11

[10] Ebisawa, K., et al. 2008, PASJ, 60, S223

[11] Matsumoto, H., et al. 2007, PASJ, 59, S199

[12] Bamba, A., et al. 2007, PASJ, 59, S209

[13] Mattana, F., et al. 2009, ApJ, 694, 12 